\title{
Reconstructability of Epistatic Functions
}

\author{
Martin Zwick ${ }^{1 *}$, Joe Fusion' ${ }^{1}$ and Beth Wilmot ${ }^{2}$ \\ Correspondence: zwick@pdx.edu \\ 'Systems Science Graduate Program, Portland State University, Portland OR 97207-0751 \\ ${ }^{2}$ Division of Bioinformatics and Computational Biology, Department of Medical Informatics and Clinical Epidemiology and Oregon Clini- \\ cal and Translational Research Institute, Oregon Health \& Sciences University, 3181 S.W. Sam Jackson Park Rd., Portland, $97239-3098$.
}

\begin{abstract}
Background: Reconstructability Analysis (RA) has been used to detect epistasis in genomic data; in that work, even the simplest RA models (variable-based models without loops) gave performance superior to two other methods. A follow-on theoretical study showed that RA also offers higher-resolution models, namely variable-based models with loops and state-based models, likely to be even more effective in modeling epistasis, and also described several mathematical approaches to classifying types of epistasis.

Methods: The present paper extends this second study by discussing a non-standard use of RA: the analysis of epistasis in quantitative as opposed to nominal variables; such quantitative variables are, for example, encountered in genetic characterizations of gene expression, e.g., eQTL data. Three methods are investigated for applying variable- and state-based RA to quantitative dependent variables: (i) k-systems analysis, which treats continuous function values as pseudofrequencies, (ii) b-systems analysis, which derives continuous values from binned DVs using expected value calculations, and (iii) u-systems analysis, which treats continuous function values as pseudo-utilities subject to a lottery. These methods are demonstrated and compared on synthetic data.

$\underline{\text { Results: }}$ The three methods of k-, b-, and u-systems analyses, both variable-based and state-based, are then applied to a published SNP dataset. A preliminary search is done with b-systems analysis, followed by more refined $\mathrm{k}$ - and $\mathrm{u}$-systems searches. The analyses suggest candidates for epistatic interactions that affect the level of gene expression. As in the synthetic data studies, state-based RA is more powerful than variable-based RA.

Conclusions: While the previous RA studies looked at epistasis in nominal (or discretized) data, this paper shows that RA can also analyze epistasis in quantitative expression data without discretizing this data. Since RA can also model epistasis in frequency distributions and detect linkage disequilibrium, its successful application here also to continuous functions shows that it offers a flexible methodology for the analysis of genomic interaction effects.

Keywords: epistasis, gene-gene interactions, gene expression, eQTL, Reconstructability Analysis, information theory, graphical models, OCCAM, bioinformatics, k-systems analysis, u-systems analysis, function decomposition, state-based modeling.
\end{abstract}

\section{Background}

Reconstructability Analysis (RA) is a modeling methodology developed in the systems science community [1-6] based on the work of Ashby [7]. It has recently been used to detect epistasis in genomic data [1], where its performance was superior to multifactor dimension reduction and neural nets. Only the simplest RA models were used, namely variable-based models without loops, so the potential value of RA for studying epistasis was not fully explored. A follow-on paper [2] showed (a) that epistasis can be modeled by RA even more effectively with more refined variable-based models with loops and with state-based models (which nearly always have loops), (b) that in classifying epistasis one can use multiple rather than single models, and (c) that the RA classification of types of epistasis can be augmented with structures from other graphical modeling techniques.
This second [2] study still did not examine all the ways that RA can be used to study epistasis. In particular, it did not consider the use of RA to analyze epistasis affecting quantitative as opposed to nominal measures, as is encountered in the study of gene expression, e.g., in eQTL data. This paper sets out the theory for such non-standard use of RA, showing with examples how analysis is done. Systematic testing on simulated data or analysis of real data for the purpose of articulating specific hypotheses about epistatic interactions are tasks left for the future. The aim of this paper is to explain and further develop RA methodology for analyzing functions, and thus lay the groundwork for such future investigations.

\section{Methods \\ Methodological distinctions}

RA is a fusion of information theory and graph theory developed

(C) 2012 Zwick et al: licensee Herbert Publications Ltd. This is an Open Access article distributed under the terms of Creative Commons Attribution License (http://creativecommons.org/licenses/by/3.0). This permits unrestricted use, distribution, and reproduction in any medium, provided the original work is properly cited. 
Table 1. Methodological distinctions for RA

\begin{tabular}{|c|c|}
\hline \multirow[t]{4}{*}{ 1. DATA TYPE } & frequency or probability distribution \\
\hline & function \\
\hline & explicit function \\
\hline & sample data \\
\hline \multirow[t]{4}{*}{ 2. MODEL TYPE } & variable-based (VB) \\
\hline & without loops \\
\hline & with loops \\
\hline & state-based (SB) (usually has loops) \\
\hline \multirow[t]{4}{*}{ 3. SYSTEM TYPE } & directed system (input/output distinction) \\
\hline & deterministic \\
\hline & non-deterministic \\
\hline & neutral system (no i/o distinction) \\
\hline \multirow[t]{3}{*}{ 4. REPRESENTATION } & b-systems (distribution or function) \\
\hline & k-systems (function) \\
\hline & u-systems (function) \\
\hline \multirow[t]{2}{*}{ 5. COMPUTATIONAL TASK } & search (exploratory) over models \\
\hline & fit, test (confirmatory), or use one model \\
\hline \multirow[t]{2}{*}{ 6. COMPOSITION ALGORITHM } & maximum entropy (standard) composition \\
\hline & Fourier composition \\
\hline
\end{tabular}

within the systems science community that partially overlaps other 'graphical models' methods that are better known, such as log-linear modeling and Bayesian networks. Table 1 summarizes various distinctions within RA methodology, and reviews of this methodology are available $[\mathbf{8 , 9}]$. Because these distinctions are not completely orthogonal, their discussion below does not simply follow the table sequentially, but the table is still a useful organizing framework. The first distinction points to the purpose of this paper: to explore the use of RA to analyze functions; but distributions are also discussed as part of necessary preliminaries. All the alternatives within the other methodological distinctions are relevant to the analysis of functions.

\section{Model types}

In standard uses of RA, both input and output variables (independent and dependent variables) are nominal with an arbitrary number of states. The input variables might be SNPs, for example, and an output variable might be the presence or absence of disease, or its surrogate, case vs. control. To illustrate how RA is applied to epistasis, let the input variables be $A, B, C$, etc. and the output variable $Z$, and let the epistatic interaction be between $A, B$, and $Z$, with $C$ having no relation to $Z$. This is captured in model $m_{1}=A B C: A B Z$, which is a variablebased model without loops, the only type of RA model used in the [1] study. This model has two relations, $A B C$ and $A B Z$, the colon ":" meaning "independent of." The first relation allows for but does not necessarily assert association between the input variables. The second is the epistatic relation, which asserts that $A$ and $B$ jointly predict $Z$. In all models, the order of relations and the order of variables within a relation are arbitrary, e.g., $A B C: A B Z$ is the same as $Z A B: C B A$.

Epistasis might be analyzed with more complex models, namely those with loops. Consider the variable-based model with a loop: $m_{2}=A B C: A Z: B Z$. The loop in this model is the cycle of interactions between $A$ and $B$ (within $A B C$ ), $B$ and $Z$, and $Z$ and $A$. The first relation again allows association between input variables; there are now two predicting relations: one in which $A$ predicts $Z$, and the other in which $B$ predicts $Z$. These two interactions are integrated with the maximum entropy principle, but there is no three-way interaction effect, so epistasis in $\mathrm{m}_{2}$ is of a weaker type than what exists in $m_{1}$ [2]. A more complex way to analyze epistasis is to use state-based models. Consider, for example, a state-based model $m_{3}=A B C: Z: A_{1} B_{1} Z: A_{2} B_{3} Z_{1}$ (which has a loop), where subscripts indicate particular states of the indicated nominal variables. The first relation again allows for association between input variables. The $Z$ component imposes the marginal distribution of the output variable. The third and fourth relations represent the power of the specific state $A_{1} B_{1}$ to predict $Z$ and an anomalously high or low probability of the $A_{2} B_{3} Z_{1}$ state. Variable-based models with loops and state-based models can more effectively characterize epistasis than variable-based models without loops because they make finer discriminations. Though their structures are more complex, they make more economical use of their degrees of freedom [2].

Three RA steps: projection, composition, evaluation In standard variable-based or state-based $R A$, the data is usually an observed frequency distribution $f(A B C Z)=N$ $p(A B C Z)$, where $N=$ sample size; or the data could be given as a probability distribution $p(A B C Z)$ without any sample size. $p(A B C Z)$ means the distribution $p\left(A_{i^{\prime}} B_{j^{\prime}} C_{k^{\prime}}, Z_{1}\right)$, for all $i, j, k, l$; the short notation will generally be used except where the long notation is needed for clarity. This is for informationtheoretic (or probabilistic) RA. There also exists a set-theoretic ("possibilistic") RA where the data is a set-theoretic relation or mapping; this type of RA is of limited interest for studying epistasis, so this methodological distinction is not included in Table 1.

Assume as before that $Z$ depends on $A$ and $B$ but not C. An RA model (e.g., $\mathrm{m}_{1}, \mathrm{~m}_{2}$, or $\mathrm{m}_{3}$ ) stipulates a calculated $A B C Z$ distribution, $p_{m}(A B C Z)$, that is generated from the projections of the data specified by the relations present in the model. The first step in constructing a model is projection, in which these relations are calculated from the data. In the composition step that follows, the relations are integrated using maximum entropy to obtain the calculated distribution for the model. For example, for data $A B C Z$ and model $A B C: A B Z$, the projection step calculates $A B C$ and $A B Z$, and the composition step joins these relations into the calculated $p_{A B C: A B Z}(A B C Z)=p(A B C) p(A B Z) / p(A B)$. For models with loops, however, composition is not algebraic, but must be iterative. What composition does is generate a calculated distribution that is as uniform as possible (has maximum entropy) as long as its calculated projections for 
the relations in the model agree with the corresponding projections of the data. As indicated in Table 1, there is a composition algorithm that is an alternative to maximum entropy, and which has been tested in a preliminary way [10], but this algorithm hasn't been implemented in the RA software used in this project, and isn't considered in this paper.

The evaluation step assesses model goodness, which depends on (i) the difference between the model distribution and some reference distribution and (ii) the degrees of freedom of the model relative to that reference. The reference model is usually either the data, $A B C Z$, or the independence model, $A B C: Z$, in which no input is associated with the output. For "neutral systems," discussed below, the reference is instead sometimes the uniform distribution. If the reference is the data, one wants the calculated $p_{m}$ to agree with the observed p. The difference between these two distributions (the error) is usually given in terms of the difference between calculated and observed Shannon entropies of these distributions, $H_{m}(A B C Z)-H(A B C Z)=T_{m}$, where $T_{m}$ is "transmission," also known as the Kullback-Leibler distance. If the reference is the independence model, one wants the calculated $p_{m}$ to differ as much as possible from a calculated $\mathrm{p}_{\text {ind }}$ as long as the difference is significant statistically, so that the model captures a maximal fraction of the information (I) in the data. Model goodness with respect to difference from the reference distribution is often tabulated as $\% \mathrm{l}=100\left(\mathrm{H}_{\text {ind }}-\mathrm{H}_{\mathrm{m}}\right) /\left(\mathrm{H}_{\text {ind }}\right.$ $-\mathrm{H}_{\text {data }}$ ) where ind=independence; when the reference is the uniform distribution, \%l $=100\left(\mathrm{H}_{\text {uniform }}-\mathrm{H}_{\mathrm{m}}\right) /\left(\mathrm{H}_{\text {uniform }}-\mathrm{H}_{\text {data }}\right)$.

\section{Model use; neutral systems}

Model $m$ is used by obtaining the conditional distribution $p_{m}(Z \mid A B)$, from $p_{m}(A B C Z)$, which allows one to predict $Z$ from $A$ and $B$. Because $Z$ is nominal in standard $R A$, using this conditional distribution for prediction is doing a classification. Here, RA is closely related (but not necessarily completely equivalent) to log-linear methods, logistic regression, and Bayesian networks. The presence of a sample size allows models and their predictions to be tested for statistical significance.

So far, discussion has concerned "directed" systems, which have inputs and at least one output. RA also includes the analysis of "neutral systems," where inputs and outputs are not distinguished; in effect, all variables are inputs (or outputs). For the analysis of quantitative functions, one RA representation - "k-systems" analysis - uses neutral systems; the two other RA representations - "b-systems" and "u-systems" analysis - use directed systems. Neutral systems can also be used to detect association between inputs, i.e., linkage disequilibrium among SNPs.

\section{Software}

RA used in this study is implemented in the program OCCAM $[11,12]$, named after "Organizational Complexity Computation and Modeling" and the famous razor (principle of economy) sometimes spelled this way. OCCAM is web-accessible, and available for use by contacting the author (Zwick). State-based searches reported in this paper were done with an auxiliary program [13], but state-based searches have recently been incorporated into OCCAM.

\section{Data}

Consider data where $Z$ is not nominal but quantitative (continuous), as occurs for example in measures of gene expression, and distinguish two types of data: (a) the data is given as an explicit function of nominal inputs $A, B$, and $C$, i.e., as $Z_{i j k}=f\left(A_{i^{\prime}} B_{j^{\prime}} C_{k}\right)$, and $(b)$ it is sample data consisting of input and output values for $\mathrm{N}$ cases, $\{\mathrm{A}(\mathrm{C}), \mathrm{B}(\mathrm{C}), \mathrm{C}(\mathrm{C}), \mathrm{Z}(\mathrm{c})\}, \mathrm{C}=1$ to $\mathrm{N}$, where $A(c)$ is some nominal $A$ value, and similarly for $B$ and $C$.

An explicit function is a simple mapping of nominal arguments onto real values, not a sample of input and output values where the same input might be observed to have multiple output values. If $f\left(A_{i^{\prime}}, B_{j^{\prime}} C_{k}\right)$ is penetrance, i.e., the probability of disease given the inputs, one can regard it as the conditional probability $p\left(Z_{1} \mid A_{i}, B_{j}, C_{k}\right)$, where $Z$ is a binary output with $Z_{0}$ being the non-diseased state and $Z_{1}$ being the diseased state. By thus adding a nominal output variable, the data is amenable to standard probabilistic analysis. If, however, $f$ is given as an arbitrary function, one does not have a probability distribution. The problem is no longer one of classification, but is the modeling of a continuous function. Log-linear methods and Bayesian networks are not applicable, but RA, in its k-systems or u-systems forms, can still be used.

In sample data, input states can repeat with different function values. One can bin (discretize) the output $Z$ values, and thus convert the data into a probability distribution to which standard RA (and log-linear methods and Bayesian networks) apply. Unless there is a binning scheme standard for the type of data being examined, a number of bins is chosen, and bin boundaries are assigned to equalize, as much as possible, the number of data points falling in each bin. ("Equal sampling" binning makes more efficient use of the data than "equal interval" binning.) A reasonable minimum default for the number of bins is three, since this allows the detection of nonlinear effects (which two bins would not). If sample size permits, more bins for $Z$ will give greater precision in calculated $Z$ values for the $b$-systems approach. In the results reported below on real data, four bins were used. Input variables that are continuous must also be binned. An input that in preliminary calculations is highly predictive can be rebinned with more bins; and one could select a number of bins that optimizes the reduction per input bin of the entropy of $Z$ [14]. In general, the number of bins assigned to all the variables is a "scarce resource", since the complexity of models that can be considered is limited, for any sample size, by the cardinalities of the variables.

The sample size allows one to calculate statistical significance, and quantitative $Z$ predictions can be recovered by a simple expected value calculation as discussed below. 
Since one is analyzing a distribution where input states can have different binned output values, the problem is stochastic. This approach is referred to as "b-systems" analysis ("b" for binning). Or, one can convert sample data to an explicit function by averaging the $f(c)$ values for each $\left(A_{i}, B_{i}, C_{k}\right)$ input state; the data is then the function $Z_{i j k}=f_{a v e}\left(A_{i}, B_{i}, C_{k}\right)$, which can be modeled with either k-systems or u-systems analysis, as explained below. In doing this, sample size information is discarded, and the analysis is non-statistical and deterministic

For both explicit functions and sample data, an important issue is whether or not function values are available for the complete input space. It is assumed in this paper that the data provides a fully defined function. How well the $b-, k-$, and u-systems approaches reconstruct partially defined functions requires a separate study.

\section{b-systems analysis}

The b-systems approach is just standard RA with one additional calculation that obtains a continuous predicted value for the output variable. The continuous prediction of $Z$ for any input state is obtained from the conditional distribution $p\left(Z_{\text {, }}\right.$ $\left|A_{i^{\prime}} B_{j^{\prime}} C_{k}\right|$ by calculating $Z^{\prime}$ 's expected value for that input state by summing over bins. Each bin, $b$, has assigned to it some continuous value, $Z_{b}$, which could be (i) the midpoint of the range of $Z$ values that define this bin or - preferably - (ii) the mean or (iii) the mode of the $Z$ values that were discretized to this bin. (Bin values can be assigned in OCCAM using its

Table 2. Hallgrímsdottir and Yuster (HY) data

$\mathrm{f}$ is the original function (data). $\mathrm{f}_{\mathrm{k} 6}-\mathrm{f}_{\mathrm{k} 8}$ are functions reconstructed from state- and variable-based $\mathrm{k}$-systems models in Table 3. $\mathrm{f}_{\mathrm{u} 6}-\mathrm{f}_{\mathrm{u} 9}$ are functions reconstructed from state- and variable-based u-systems models in Table 5, further below.

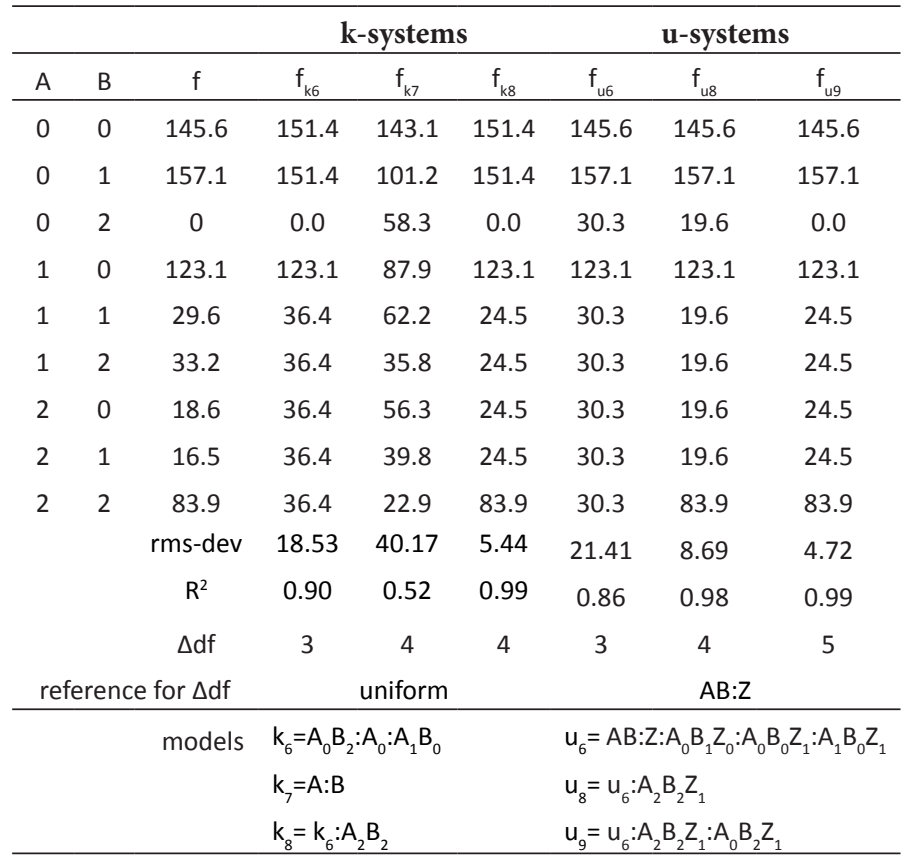

rebinning option.) $Z^{\prime}$ s expected value is $\left\langle Z_{i i j}\right\rangle=\Sigma_{b} Z_{b} p\left(Z_{b} \mid\right.$ $\left.A_{i}, B_{j}\right)$. The entropy of this conditional distribution for each input state is a measure of the confidence that should be associated with this expected $Z$ value; one can also calculate a variance for this expected value.

Binned gene expression data were used in a preliminary RA study [15] but expected value predictions were not examined there. That study employed only simple variable-based models without loops, but it successfully replicated and extended SNP-gene expression associations in a public dataset [16].

\section{k-systems analysis}

The use of "k-systems" analysis to compress (decompose) functions of nominal variables was first proposed by Jones [17], who joined it to state-based analysis, but the k-systems approach can also be used with variable-based analysis [18]. This approach applies to data that is an explicit function of nominal variables. If sample data is given, it is converted to an explicit function by averaging function values for every input state. The within-input-state variation in the function value and the frequencies of the input states are ignored.

The central idea in k-system analysis is to linearly scale the function (its average value for every input state) so it can be treated as a pseudo-probability, do standard reconstructability analysis on this distribution, and then do an inverse scaling of reconstructed pseudo-probabilities to get an approximation to the original function. Jones called the scaled function " $k$ " in honor of George Klir, one of the pioneers of RA, hence the name "k-systems analysis." Here the scaled function is called $p$, because it is treated as if it were a probability. Scaling is done as follows:

$p(A B C)=a f(A B C)+b$, where $a$ and $b$ are constants, such that $0 \leq p(A B C) \leq 1$ for all states of $A B C$, and $\Sigma \Sigma \Sigma p(A B C)=1$

$b$ is chosen to bring negative values of $f$ to zero or to $a$ slightly positive value (negative values are not relevant to gene

Table 3. State- and variable-based k-systems analysis of $\mathrm{HY}$ data $\mathrm{H}=$ Shannon entropy, $\% \mathrm{I}=\%$ information, $\mathrm{df}=$ degrees of freedom. Variable-based models are in italics. Models are neutral systems.

\begin{tabular}{llccl}
\hline & Model & $\mathbf{H}$ & $\mathbf{\% l}$ & $\mathbf{\Delta d f}$ \\
\hline 10 & $A B$ & 2.596 & $100.0 \%$ & 8 \\
9 & $\mathrm{~A}_{0} \mathrm{~B}_{2}: \mathrm{A}_{0}: \mathrm{A}_{1} \mathrm{~B}_{0}: \mathrm{A}_{2} \mathrm{~B}_{2}: \mathrm{A}_{2}$ & 2.597 & $99.8 \%$ & 5 \\
8 & $\mathrm{~A}_{0} \mathrm{~B}_{2}: \mathrm{A}_{0}: \mathrm{A}_{1} \mathrm{~B}_{0}: \mathrm{A}_{2} \mathrm{~B}_{2}$ & 2.607 & $98.2 \%$ & 4 \\
7 & $A_{1}: \mathrm{B}_{0}$ & 2.981 & $32.9 \%$ & 4 \\
6 & $\mathrm{~A}_{0} \mathrm{~B}_{2}: \mathrm{A}_{0}: \mathrm{A}_{1} \mathrm{~B}_{0}$ & 2.681 & $85.2 \%$ & 3 \\
5 & $\mathrm{~A}_{0} \mathrm{~B}_{2}: \mathrm{A}_{0}$ & 2.795 & $65.3 \%$ & 2 \\
4 & $A$ & 3.069 & $17.6 \%$ & 2 \\
3 & $\mathrm{~B}_{2}$ & 3.082 & $15.3 \%$ & 2 \\
2 & $\mathrm{~A}_{0} \mathrm{~B}_{2}$ & 3.000 & $29.6 \%$ & 1 \\
1 & Uniform & 3.170 & $0.0 \%$ & 0 \\
\hline
\end{tabular}


expression, but this discussion applies to functions in general); $a$ is then determined by the normalization condition that the probabilities sum to 1 . The scaled $p(A B C)$ is then subjected to standard neutral system RA. Suppose the variable-based model $A B: B C$ with its calculated distribution, $p_{A B: B C}(A B C)$, is recommended by the analysis. The reconstructed function is obtained by the inverse scaling, i.e.,

$$
f_{A B: B C}(A B C)=\left(p_{A B: B C}(A B C)-b\right) / a
$$

One can as well use state-based modeling to decompose an observed $p(A B C)$ and obtain a calculated $p_{m}(A B C)$. Jones joined scaling-decomposition-rescaling with state-based modeling, but these two procedures are separable.

The k-systems RA approach can be compared to the analysis of variance without replication, also used to study the association between nominal inputs and a quantitative output that is unique for each input state. Jones [19] and Gouw and Jones [20] discuss these alternative methodologies and argue for the merits of their approach, which differs from multivariate experimental design methods in making no assumptions about the functional form of the relation between inputs and output or of the distribution of errors. However, k-systems analysis is not statistical, and does not offer confidence estimates, so its results need to be subjected to cross-validation studies in which test data assess the generalizability of models obtained from training data.

The following tables show the k-systems analysis of a function of two variables, and the efficacy of state- as compared to variable-based modeling. Table 2 is data from Hallgrímsdottir and Yuster [21], who classify types of twolocus epistasis. Since the lowest function value is 0 , the function needs only ratio scaling by a to convert it to a pseudoprobability. A and B have cardinality 3 , so the data's degrees of freedom (df) is 8 , assuming knowledge of the average $f$.

Table 3 shows the results of both state- and variable-based k-systems analysis. The results show that the function can be represented by many fewer degrees of freedom if state-based models are used. State-based model \#6, $A_{0} B_{2}: A_{0}: A_{1} B_{0}$, with
$\Delta d f=3$, has one fewer degree of freedom than the variablebased model \#7, A:B; yet model \#6 captures $85.2 \%$ of the information in the data, while model \#7 captures only $32.9 \%$. State-based model \#8, with the same $\Delta \mathrm{df}=4$ as model \#7, captures $98.2 \%$, almost three times the information of model \#7. Similarly, for $\Delta d f=2$, state-based model $\# 5, A_{0} B_{2}: A_{0}$ has about four times the information of variable-based models \#3 and \#4. The reconstructed functions for models \#6, \#7, and \#8 are shown in Table 2 where closeness of these functions to $f$ is indicated by rms-dev and $R^{2}$. In Table 3, closeness of the reconstructed functions to $f$ is given by entropy-based $\%$ l.

Since k-systems analysis is non-statistical - there is no sample size that can be meaningfully used - the choice of a particular model and its reconstructed function must be made on the basis of user-provided criteria, based only on $\% 1$ and $\Delta \mathrm{df}$. In the present case, models \#6 and \#8 are plausible candidates, but \#7 is not. State-based analysis of continuous output functions is more powerful than variable-based analysis.

\section{u-systems analysis}

The u-systems approach, like k-systems analysis, applies to functions defined uniquely by their inputs. The essential idea underlying this approach is borrowed from expected value calculations in decision theory $[13,18]$ : any utility value can be regarded as the expected value of an appropriate lottery. Similarly, any function value can be generated as an expected value of an appropriate probability distribution. Thus, for example, if we wish our expected value to equal the actual function value, i.e.,

$$
\left\langle Z>_{i j}=p\left(Z_{0} \mid A_{i} B_{j}\right) Z_{0}+p\left(Z_{1} \mid A_{i} B_{j}\right) Z_{1}=f\left(A_{i} B_{j}\right),\right.
$$

this can be accomplished by setting the representative values for the bins to the minimum and maximum values of the function, i.e., $\mathrm{Z}_{0}=\mathrm{f}_{\min }$ and $\mathrm{Z}_{1}=\mathrm{f}_{\max }$ and by defining

$$
p\left(Z_{1} \mid A_{i} B_{j}\right)=\left(f\left(A_{i} B_{j}\right)-f_{\text {min }}\right) /\left(f_{\text {max }}-f_{\text {min }}\right) ; p\left(Z_{0} \mid A_{i} B_{j}\right)=1-p\left(Z_{1} \mid A_{i} B_{j}\right) .
$$

Table 4. u-systems form of Hallgrímsdottir and Yuster (HY) data

\begin{tabular}{ccccc}
\hline $\mathbf{A}$ & $\mathbf{B}$ & $\mathbf{f}$ & $\mathbf{p}\left(\mathbf{Z}_{0} \mid \mathbf{A B}\right)$ & $\mathbf{p}\left(\mathbf{Z}_{1} \mid \mathbf{A B}\right)$ \\
\hline 0 & 0 & 145.6 & 0.072 & 0.927 \\
0 & 1 & 157.1 & 0.000 & 1.000 \\
0 & 2 & 0 & 1.000 & 0.000 \\
1 & 0 & 123.1 & 0.216 & 0.783 \\
1 & 1 & 29.6 & 0.810 & 0.189 \\
1 & 2 & 33.2 & 0.792 & 0.207 \\
2 & 0 & 18.6 & 0.882 & 0.117 \\
2 & 1 & 16.5 & 0.891 & 0.108 \\
2 & 2 & 83.9 & 0.072 & 0.927 \\
\hline
\end{tabular}

Table 5. State- and variable-based u-systems analysis of $\mathrm{HY}$ data Variable-based models are in italics. Models are directed systems.

\begin{tabular}{clccc}
\hline & Model & H & \%1 & ddf \\
\hline 10 & $A B Z$ & 3.679 & $100.0 \%$ & 8 \\
9 & $A B: Z: A_{0} B_{1} Z_{0}: A_{0} B_{0} Z_{1}: A_{1} B_{0} Z_{1}: A_{2} B_{2} Z_{1}: A_{0} B_{2} Z_{1}$ & 3.684 & $99.0 \%$ & 5 \\
8 & $A B: Z: A_{0} B_{1} Z_{0}: A_{0} B_{0} Z_{1}: A_{1} B_{0} Z_{1}: A_{2} B_{2} Z_{1}$ & 3.708 & $93.9 \%$ & 4 \\
7 & $A B: A Z: B Z$ & 4.004 & $31.9 \%$ & 4 \\
6 & $A B: Z: A_{0} B_{1} Z_{0}: A_{0} B_{0} Z_{1}: A_{1} B_{0} Z_{1}$ & 3.767 & $81.4 \%$ & 3 \\
5 & $A B: Z: A_{0} B_{1} Z_{0}: A_{0} B_{0} Z_{1}$ & 3.874 & $59.0 \%$ & 2 \\
4 & $A B: A Z$ & 4.078 & $16.3 \%$ & 2 \\
3 & $A B: B Z$ & 4.090 & $13.8 \%$ & 2 \\
2 & $A B: Z: A_{0} B_{1} Z_{0}$ & 4.007 & $31.2 \%$ & 1 \\
1 & $A B: Z$ & 4.156 & $0.0 \%$ & 0 \\
\hline
\end{tabular}


For the $\left(A_{i}, B_{i}\right)$ value for which the function is maximum, $p\left(Z_{1}\right)$ $=1$ and $p\left(Z_{0}\right)=0$; for the $\left(A_{i}, B_{j}\right)$ value for which the function is minimum, $p\left(Z_{1}\right)=0$ and $p\left(Z_{0}\right)=1$.

Applying this approach to the Hallgrímsdottir and Yuster [21] data of Table 2 gives the $p\left(Z_{0} \mid A_{i} B_{j}\right)$ and $p\left(Z_{1} \mid A_{i} B_{j}\right)$ values shown in Table 4. To get $A B Z$ data, these conditional probabilities, $p(Z \mid A B)$, must be multiplied by some $p(A B)$. Since the HallgrímsdottirYuster data is an explicit function, $p(A B)$ is taken to be a constant.

In applications where sample data is available, one has frequencies for the different input states. In k-systems analysis, these are not usable, but in u-systems analysis, one could multiply $p(Z \mid A B)$ by the input state probability, $p(A B)$. This does a weighting of function values; alternatively, weights based on variances of function values for different input states might be used. Here no weighting has been done. The results of variable- and state-based u-systems analysis of the HY data are provided by Table 5; the reconstructed functions for state-based models \#6, \#8, and \#9 are given in Table 2.

Once again, results show that state-based analysis is more powerful than variable-based analysis. This is indicated by the higher information content of state-based model \#5 compared to the information contents of variable-based models \#4 and \#3, all of which have $\Delta \mathrm{df}=2$; also by the higher information content of state-based model \#8 compared to that of variable-based model $\# 7$, both of which have $\Delta \mathrm{df}=4$.

The $\mathrm{u}$-systems analysis of Table 5 resembles but is still different from the k-systems analysis of Table 3. Table 6 shows the sequence of states added in these two searches. The analytical relationship between these two representations and the arguments for using one versus the other are under investigation. Still, the results of these two different mathematical approaches are in rough agreement.

Comparing the rms-deviations and $\mathrm{R}^{2} \mathrm{~s}$ given in Table 2 for the u-systems reconstructed functions (for models \#6, \#8, and \#9) to the same measures given in Table 2 for the k-systems reconstructed functions (for models \#6 and \#8) suggests that, on the HY data, k-systems reconstruction performs slightly better than u-systems reconstruction. However, a general conclusion about the relative merits of these two approaches to reconstructing continuous functions must await comparative studies on more datasets.

\section{Comparing b-, k-, and u-systems analyses}

Three different approaches are thus available for the analysis of functions such as might be obtained from gene expression data: b-systems analysis, which is a slight extension of standard probabilistic analysis, which requires that function values be binned; k-systems analysis, which rescales the function so it can be treated as a pseudo-probability; and u-systems analysis, which treats the function value as the expected value of a lottery. The salient properties of these approaches are summarized in Table 7.

\section{Comments:}

1. The input-output relation in u-systems analysis is deterministic in that only the average function value for any input state is used; however, it is stochastic in that the analysis itself is probabilistic (the output has two states).

2. The use of input state repeats is possible in u-systems analysis in that the probabilities of input states or the variances of their function values could be used to weight the conditional probabilities of the output states.

3. Analysis with b-systems is statistical in that likelihood ratio values, $\mathrm{p}$-values, and information measures (e.g., the Akaike and Bayesian Information Criteria) are relevant to the choice of model. These measures are not relevant and thus cannot be used for model selection for $\mathrm{k}$ - or u-systems, since a sample size is either unavailable or irrelevant.

4. The use of exact function values in k-systems and $\mathrm{u}$-systems is an advantage since artifacts introduced by binning are avoided; $b$-systems analysis may give results that are sensitive to the exact procedures and parameters used for discretization.

5. The range limits of a u-systems reconstructed function can be extended by choosing bin values beyond the minimum and maximum function values in the data. By contrast, the range of a b-systems reconstructed function is limited by those bins populated by the data, while the range of a k-systems reconstructed function is not inherently limited.
Table 6. Sequence of added states in $\mathrm{k}$ - and u-systems analysis of HY data.

\begin{tabular}{ccc}
\hline & $\mathbf{k}$ & $\mathbf{u}$ \\
\hline 5 & $\mathrm{~A}_{2}$ & $\mathrm{~A}_{0} \mathrm{~B}_{2} \mathrm{Z}_{1}$ \\
4 & $\mathrm{~A}_{2} \mathrm{~B}_{2}$ & $\mathrm{~A}_{2} \mathrm{~B}_{2} \mathrm{Z}_{1}$ \\
3 & $\mathrm{~A}_{1} \mathrm{~B}_{0}$ & $\mathrm{~A}_{1} \mathrm{~B}_{0} \mathrm{Z}_{1}$ \\
2 & $\mathrm{~A}_{0}$ & $\mathrm{~A}_{0} \mathrm{~B}_{0} \mathrm{Z}_{1}$ \\
1 & $\mathrm{~A}_{0} \mathrm{~B}_{2}$ & $\mathrm{~A}_{0} \mathrm{~B}_{1} \mathrm{Z}_{0}$ \\
\hline
\end{tabular}

Table 7. Comparing the b-, k-, and u-systems approaches Preferred values of attributes are in bold.

\begin{tabular}{|c|c|c|c|}
\hline attribute & b-systems & k-systems & u-systems \\
\hline system type & directed & neutral & directed \\
\hline presence of loops & more common & less common & more common \\
\hline input-output relation & stochastic & deterministic & deterministic ${ }^{1}$ \\
\hline use of input state repeats & yes & no & possible $^{2}$ \\
\hline statistical tests ${ }^{3}$ & yes & no & no \\
\hline use of exact function values & no (binned) $)^{4}$ & yes (of average) & yes (of average) \\
\hline reconstruction range limited & yes (by bins) & no & yes but arbitrary ${ }^{5}$ \\
\hline
\end{tabular}




\section{Results: A real data example}

In a preliminary study [22], RA was used to analyze data on states for 75 SNPs in eight genes and expression values for these genes. The expression values were binned, but the study aimed only at detecting SNPs that predicted expression, so continuous expression functions of the SNP states were not also generated. This study used only variable-based models without loops, and its primary results were models with only one predicting SNP. This simple analysis detected associations between SNP alleles and binned gene expression values, replicating and extending SNP-gene expression associations in a public data set [16]. Only the data from SNPs and expression values from the published positive cis-acting (local) SNP-gene expression associations were included. RA identified all but two cis-acting SNPs (false negative rate = 2.7\%) and detected additional cis-acting SNPs.

To further explore the use of RA for quantitative functions, continuous gene expression values were generated from this data in a two-step process: the first step did a coarse search among models using b-systems analysis, the second step did a finer analysis of the results of the first step using the $k$ - and u-systems approaches.

Step 1: b-systems analysis. A b-systems search through the space of models, including models with loops, was done with OCCAM. The expression values were discretized into four bins. Before doing this search, cases with missing data for salient variables were eliminated from the data, since OCCAM currently treats missing data as an additional state of the affected variable, and this extra state (a) can introduce artifacts into the analysis and (b) adds complexity to the model which raises the threshold for statistical significance. The original data had $\mathrm{N}=193$; after case elimination, $\mathrm{N}=83$, yet despite this drop in sample size, more models were significant because models were simpler. The search results are shown in Table 8. The search was done for three levels of complexity; at each level, the best 25 models are selected from the larger set of models which were generated by adding one relation to the best 25 models of the preceding lower level.

A promising model from the results of this search was selected for more detailed $\mathrm{k}$ - and $\mathrm{u}$-systems analyses. The selection was based on the OCCAM results summary, which tabulates "best models" using different model selection criteria. The most conservative of these criteria is (i), the Bayesian Information Criterion (BIC), which favors simple (low $\Delta \mathrm{df}$ ) models. Just as in the Wilmot [22] study, where BIC favored loopless models having only one SNP predictor, the search results identified a set of five equivalent models, of form $\mathrm{IV}: \mathrm{XZ}$, where $\mathrm{Z}$ is the binned expression value for gene KIF1B, $\mathrm{IV}$ is a component that contains all the independent variables (the 75 SNPs), and $X$ is any one of the $\{N, O, P, Q, R\} S N P s$, all of which are in KIF1B and in strong linkage disequilibrium (LD) with one another. IV:OZ was arbitrarily chosen to represent this set of models.

A less conservative criterion (ii) was also used that selects the model with the highest \%l that is statistically significant $(p<.05)$ relative to a reference of independence, and for

Table 8. b-systems variable-based model search for gene KIF1B

Models are directed systems. "(n models)" means n equivalent models, not listed. LR and p are likelihood ratio and significance relative to the IV: $\mathrm{Z}$ reference; $\mathrm{p}_{\text {incr }}$ is incremental significance relative to the immediate model 'ancestor'. Good models have low $\mathrm{H}, \Delta \mathrm{df}, \mathrm{p}, \mathrm{p}_{\mathrm{incr}}$ and high $\Delta \mathrm{LR}, \% \mathrm{I}, \Delta \mathrm{AIC}, \Delta \mathrm{BIC}$.

\begin{tabular}{|c|c|c|c|c|c|c|c|c|c|}
\hline Model & Level & $\mathbf{H}$ & $\Delta \mathrm{df}$ & $\Delta \mathrm{LR}$ & $\mathbf{p}$ & $\% \mathbf{I}$ & $\Delta \mathrm{AIC}$ & $\Delta \mathrm{BIC}$ & $\mathbf{p}_{\text {incr }}$ \\
\hline IV:XZ:YZ:MZ (25 models) & 3 & 7.46 & 12 & 60.3 & 0.000 & $32.7 \%$ & 36.3 & 7.1 & 0.077 \\
\hline IV:XZ:YZ (25 models) & 2 & 7.53 & 8 & 51.9 & 0.000 & $28.1 \%$ & 35.9 & 16.4 & 0.000 \\
\hline IV:XZ (5 models) & 1 & 7.66 & 4 & 36.9 & 0.000 & $20.0 \%$ & 28.9 & 19.2 & 0.000 \\
\hline IV:WZ (3 models) & 1 & 7.67 & 4 & 35.2 & 0.000 & $19.1 \%$ & 27.2 & 17.5 & 0.000 \\
\hline \multicolumn{10}{|l|}{13 other models } \\
\hline IV:YZ (4 models) & 1 & 7.86 & 4 & 14.0 & 0.007 & $7.6 \%$ & 6.0 & -3.7 & 0.007 \\
\hline $\mathrm{IV}: \mathrm{Z}$ & 0 & 7.98 & 0 & 0.0 & 1.000 & $0.0 \%$ & 0.0 & 0.0 & 0.000 \\
\hline \multicolumn{10}{|l|}{ Model selection criteria } \\
\hline \multicolumn{10}{|l|}{ i. Best Model(s) by $\Delta \mathrm{BIC}$ : } \\
\hline IV:XZ & 1 & 7.66 & 4 & 36.9 & 0.000 & 0.1999 & 28.9 & 19.2 & 0.000 \\
\hline \multicolumn{10}{|c|}{ ii. Best Model(s) by $\%$ with $\mathrm{p}<0.05$ and also all $\mathrm{p}_{\text {incr }}<0.05$ : } \\
\hline $\mathrm{IV}: X Z: Y Z$ & 2 & 7.53 & 8 & 51.9 & 0.000 & 0.2811 & 35.9 & 16.4 & 0.000 \\
\hline \multicolumn{10}{|l|}{ iii. Best Model(s) by $\triangle$ AIC: } \\
\hline $\mathrm{IV}: \mathrm{XZ}: \mathrm{YZ}: \mathrm{MZ}$ & 3 & 7.46 & 12 & 60.3 & 0.000 & $32.7 \%$ & 36.3 & 7.1 & 0.077 \\
\hline \multicolumn{10}{|c|}{ iv. Best Model(s) by $\%$ I with $p<0.05$ : } \\
\hline $\mathrm{IV}: \mathrm{XZ}: \mathrm{YZ}: \mathrm{MZ}$ & 3 & 7.46 & 12 & 60.3 & 0.000 & $32.7 \%$ & 36.3 & 7.1 & 0.077 \\
\hline
\end{tabular}


Zwick et al. Journal of Molecular Engineering \& Systems Biology 2012, http://www.hoajonline.com/journals/pdf/2050-1412-1-4.pdf

Table 9. SNPs in models selected

The SNP that represents the LD group is in bold

\begin{tabular}{ll|rl}
\hline & X SNPs & \multicolumn{2}{|l}{ Y SNPs } \\
\hline N & rs946501 & G & rs6433464 \\
O & rs9332414 & H & rs11674895 \\
P & rs17034643 & I & rs10195413 \\
Q & rs121242 & J & rs4972643 \\
R & rs12120191 & K & rs10930654 \\
& & L & rs4144329 \\
M & rs1995969 & & \\
\hline
\end{tabular}

Table 11. State-based b-systems analysis of KIF1B

Variable-based models are in italics. Models are directed systems.

\begin{tabular}{llccccc}
\hline & Model & $\mathbf{H}$ & $\mathbf{\% l}$ & $\mathbf{\Delta d f}$ & $\mathbf{p}$ & $\mathbf{p}_{\text {incr }}$ \\
\hline 7 & OGZ & 4.128 & $100.0 \%$ & 16 & 0.000 & 0.879 \\
6 & OG:Z:O $\mathrm{O}_{0}: \mathrm{O}_{2} \mathrm{Z}_{0}: \mathrm{O}_{1} \mathrm{Z}_{2}: \mathrm{O}_{0} \mathrm{G}_{0} \mathrm{Z}_{1}: \mathrm{O}_{2} \mathrm{G}_{0} \mathrm{Z}_{1}$ & 4.151 & $93.4 \%$ & 5 & 0.000 & 0.164 \\
5 & OG:Z:O $\mathrm{O}_{0} \mathrm{O}_{0} \mathrm{O}_{2} \mathrm{Z}_{0} \mathrm{O}_{1} \mathrm{Z}_{2}: \mathrm{O}_{0} \mathrm{G}_{0} \mathrm{Z}_{1}$ & 4.158 & $91.3 \%$ & 4 & 0.000 & $\mathbf{0 . 0 4 6}$ \\
4 & OG:Z:O $\mathrm{O}_{0} \mathrm{Z}_{0}: \mathrm{O}_{2} \mathrm{Z}_{0}: \mathrm{O}_{1} \mathrm{Z}_{2}$ & 4.174 & $86.8 \%$ & 3 & 0.000 & 0.026 \\
3 & OG:Z:O $\mathrm{O}_{0}: \mathrm{O}_{2} \mathrm{Z}_{0}$ & 4.193 & $81.3 \%$ & 2 & 0.000 & 0.000 \\
2 & OG:Z:O$: \mathrm{O}_{0}$ & 4.273 & $58.3 \%$ & 1 & 0.000 & 0.000 \\
1 & OG:Z & 4.475 & $0.0 \%$ & 0 & 1.000 & 1.000 \\
\hline
\end{tabular}

Table 10. b- and k-systems reconstructions for gene KIF1B $f_{b 1}-f_{b 3}$ are reconstructed functions for variable-based $b$-systems analyses, whose details are not included. $\mathrm{f}_{\mathrm{b} 4}$ and $\mathrm{f}_{\mathrm{b} 5}$ are reconstructed functions from b-systems models \#4 and \#5 in Table 11. $\mathrm{f}_{\mathrm{k} 3}$ and $\mathrm{f}_{\mathrm{k} 4}$ are for k-systems models \#3 and \#4 in Table 12

\begin{tabular}{|c|c|c|c|c|c|c|c|c|c|}
\hline \multirow[b]{3}{*}{ O } & \multirow[b]{3}{*}{ G } & \multirow[b]{3}{*}{ f } & \multicolumn{5}{|c|}{ b-systems } & \multirow{2}{*}{\multicolumn{2}{|c|}{$\begin{array}{c}\text { k-systems } \\
\text { state-based }\end{array}$}} \\
\hline & & & \multicolumn{3}{|c|}{ variable-based } & \multicolumn{2}{|c|}{ state-based } & & \\
\hline & & & $f_{b 1}$ & $f_{b 2}$ & $f_{b 3}$ & $\mathrm{f}_{\mathrm{b} 4}$ & $f_{b 5}$ & $f_{k 3}$ & $f_{k 4}$ \\
\hline 0 & 0 & 2.34 & 2.40 & 2.36 & 2.37 & 2.39 & 2.36 & 2.39 & 2.39 \\
\hline 0 & 1 & 2.41 & 2.40 & 2.41 & 2.40 & 2.39 & 2.40 & 2.39 & 2.39 \\
\hline 0 & 2 & 2.41 & 2.40 & 2.40 & 2.40 & 2.39 & 2.40 & 2.39 & 2.39 \\
\hline 1 & 0 & 2.21 & 2.22 & 2.20 & 2.19 & 2.22 & 2.22 & 2.25 & 2.25 \\
\hline 1 & 1 & 2.28 & 2.22 & 2.24 & 2.25 & 2.22 & 2.22 & 2.25 & 2.25 \\
\hline 1 & 2 & 2.25 & 2.22 & 2.19 & 2.19 & 2.22 & 2.22 & 2.25 & 2.25 \\
\hline 2 & 0 & 1.84 & 2.04 & 2.03 & 2.04 & 2.04 & 2.04 & 1.84 & 1.87 \\
\hline 2 & 1 & 1.90 & 2.04 & 2.04 & 2.04 & 2.04 & 2.04 & 1.84 & 1.87 \\
\hline \multirow[t]{6}{*}{2} & 2 & 1.76 & 2.04 & 2.03 & 2.00 & 2.04 & 2.04 & 1.84 & 1.76 \\
\hline & & rms-dev & 0.124 & 0.121 & 0.117 & 0.125 & 0.124 & 0.041 & 0.029 \\
\hline & & $\mathrm{R}^{2}$ & 0.904 & 0.913 & 0.917 & 0.905 & 0.915 & 0.970 & 0.985 \\
\hline & & $\Delta \mathrm{df}$ & 4 & 8 & 16 & 3 & 4 & 2 & 3 \\
\hline & refer & $\begin{array}{r}\text { ence for } \\
\Delta \mathrm{df}\end{array}$ & & & OG:Z & & & uni & orm \\
\hline & & models & $\begin{array}{l}b_{1}=O G: C \\
b_{4}=O G \\
b_{5}=O G\end{array}$ & $\begin{array}{l}O Z \quad b_{2}= \\
: Z: O_{0} Z_{0}: \\
: Z: O_{0}: C\end{array}$ & $\begin{array}{l}\mathrm{OG}: \mathrm{OZ}: \mathrm{GZ} \\
\mathrm{Z}_{2}: \mathrm{O}_{1} \mathrm{Z}_{2} \\
\mathrm{Z}_{2}: \mathrm{O}_{1} \mathrm{Z}_{2}: \mathrm{C}\end{array}$ & $\begin{array}{l}\mathrm{Z} \quad \mathrm{b}_{3}=\mathrm{c} \\
\mathrm{O}_{0} \mathrm{G}_{0} \mathrm{Z}_{1}\end{array}$ & & $\begin{array}{l}\mathrm{k}_{3}=0 \\
\mathrm{k}_{4}=0: 0\end{array}$ & \\
\hline
\end{tabular}

which each incremental df increase from independence is also statistically significant $\left(p_{\text {incr }}<.05\right)$. This "path-significance" criterion selected best models of the form, IV:XZ:YZ, which has a loop, where $X$ is as above and $Y$ is any one of the $\{G, H, I$, $J, K, L\}$ SNPs, all in gene OLA1 and also in strong LD with one another. IV:OZ:GZ was arbitrarily chosen to represent this set of b-systems models. The two less conservative criteria, (iii) $\mathrm{AIC}$, and (iv) cumulative but not also incremental significance, being more vulnerable to false positives, were not considered. The b-systems search thus yields two models IV:OZ and IV:OZ:GZ (and their equivalents). The SNPs represented by $X$ and $Y$ (and $M$ in IV:XZ:YZ:MZ) are listed in Table 9. That the $X$ variables are in strong $L D$ with one another, as are the $Y$ variables, is shown by an OCCAM neutral system run that looks only for associations between the SNPs (it drops the $D V) ;$ this calculation yields the $X$ and $Y$ clusters. Also, in a directed system calculation where the $D V$ is $O$ or $G$, the other SNPs in each group maximally predict $(\% \mathrm{l}=100 \%)$ their representative SNP.

Table 10 shows $f(O G)$, the average expression for the OG states in the data, and $\mathrm{f}_{\mathrm{b} 1}(\mathrm{OG}), \mathrm{f}_{\mathrm{b} 2}(\mathrm{OG})$, and $\mathrm{f}_{\mathrm{b} 3}(\mathrm{OG})$, the expected-value reconstructions of three variable-based b-systems models (detailed results not shown), with their $R^{2}$ and rms-deviations relative to $f(O G)$. This calculation only deleted cases where data was missing for $\mathrm{O}$ or $\mathrm{G}$, so here $\mathrm{N}=187$. $\mathrm{f}_{\mathrm{b} 1}$ and $\mathrm{f}_{\mathrm{b} 2}$ are the reconstructed functions for IV:OZ and IV:OZ:GZ, the best models in the search shown in Table 8 according to $\mathrm{BIC}$ and path-significance criteria, respectively. $f_{b 3}$ is the reconstructed function for a triadic variable-based interaction between $\mathrm{O}, \mathrm{G}$, and $\mathrm{Z}$. In the terminology of the earlier RA study of epistasis [2], IV:OGZ is Type 1 epistasis, the strongest type; IV:OZ:GZ is Type 2 epistasis, a weaker type. IV:OZ, having only one SNP predictor, is not epistatic at all. All three of these b-systems models agree well with the data, and the only slight improvement in $R^{2}$ (from 0.904 to 0.913 or 0.917 ) by adding $\mathrm{G}$ as a predictor (either via Type 2 or Type 1 epistasis) suggests that this addition may not be warranted, even though this addition was incrementally significant in the initial b-systems search (model IV:XZ:YZ in Table 8).

Table 10 also indicates the results of expected value reconstructions of two state-based b-systems models. The state-based analysis is shown in Table 11. State-based b-systems models are slightly better (higher $\mathrm{R}^{2}$ and/or lower $\Delta$ df) than variable-based b-systems models. Note that adding the fourth state (going from model \#4 to \#5), in bold, which indicates an epistatic interaction, is incrementally (and cumulatively) significant.

Step 2.1: k-systems analysis. Table 10 above includes models \#3 and \#4 from a k-systems analysis whose results are given below in Table 12. The first state selected (model \#2) was $\mathrm{O}_{2}$, whose average expression value is notably different from the other two $\mathrm{O}$ states. This state alone embodies $91.4 \%$ of the data. The second state selected (model \#4) was $\mathrm{O}_{0^{\prime}}$ 
Table 12. State-based k-systems analysis for KIF1B

Variable-based models are in italics. Models are neutral systems. Model \#6 is the data. The reconstructed function for models \#3 and \#4 are shown above in Table 10

\begin{tabular}{llccc}
\hline & Model & $\mathbf{H}$ & $\boldsymbol{\%} \mathbf{l}$ & $\boldsymbol{\Delta} \mathbf{d f}$ \\
\hline 6 & $\mathrm{OG}$ & 3.16090 & $100.0 \%$ & 8 \\
5 & $\mathrm{O}_{2}: \mathrm{O}_{0}: \mathrm{O}_{2} \mathrm{G}_{2}: \mathrm{G}_{0}$ & 3.16091 & $99.9 \%$ & 4 \\
4 & $\mathrm{O}_{2}: \mathrm{O}_{0}: \mathrm{O}_{2} \mathrm{G}_{2}$ & 3.16103 & $98.6 \%$ & 3 \\
3 & $\mathrm{O}_{2}: \mathrm{O}_{0}=O$ & 3.16119 & $96.8 \%$ & 2 \\
2 & $\mathrm{O}_{2}$ & 3.16168 & $91.4 \%$ & 1 \\
1 & uniform & 3.16993 & $0.0 \%$ & 0 \\
\hline
\end{tabular}

Table 13. State-based u-systems analysis for KIF1B Variable-based models are in italics. Models are directed systems. Model \#7 is the data. The reconstructed functions for models \#2 and \#3 are the same as $f_{k 2}$. and $f_{k 3}$ in Table 10.

\begin{tabular}{llccc}
\hline & Model & $\mathbf{H}$ & \%l & $\boldsymbol{\Delta d f}$ \\
\hline 7 & OGZ & 3.636 & $100.0 \%$ & 8 \\
6 & OG:Z:O $\mathrm{O}_{2}: \mathrm{O}_{1} \mathrm{Z}_{1}: \mathrm{O}_{2} \mathrm{G}_{2} \mathrm{Z}_{1}: \mathrm{O}_{0} \mathrm{G}_{0} \mathrm{Z}_{1}: \mathrm{G}_{1} \mathrm{Z}_{1}$ & 3.640 & $99.1 \%$ & 5 \\
5 & OG:Z:O $\mathrm{Z}_{1}: \mathrm{O}_{1} \mathrm{Z}_{1}: \mathrm{O}_{2} \mathrm{G}_{2} \mathrm{Z}_{1}: \mathrm{O}_{0} \mathrm{G}_{0} \mathrm{Z}_{1}$ & 3.645 & $98.2 \%$ & 4 \\
4 & OG:Z:O $\mathrm{O}_{2}: \mathrm{O}_{1} \mathrm{Z}_{1}: \mathrm{O}_{2} \mathrm{G}_{2} \mathrm{Z}_{1}$ & 3.660 & $95.2 \%$ & 3 \\
3 & OG:Z:O $\mathrm{Z}_{1}: \mathrm{O}_{1} \mathrm{Z}_{1}=\mathrm{OG}: O Z$ & 3.683 & $90.6 \%$ & 2 \\
2 & OG:Z:O $\mathrm{O}_{1}$ & 3.735 & $80.1 \%$ & 1 \\
1 & OG:Z: & 4.137 & $0.0 \%$ & 0 \\
\hline
\end{tabular}

Table 14. Sequence of added states in b-, k-, and u-systems analyses of KIF1B data

\begin{tabular}{ccrc}
\hline & $\mathbf{b}$ & $\mathbf{k}$ & $\mathbf{u}$ \\
\hline 5 & $\mathrm{O}_{2} \mathrm{G}_{0} \mathrm{Z}_{1}$ & $\mathrm{O}_{1} \mathrm{G}_{2}$ & $\mathrm{G}_{1} \mathrm{Z}_{1}$ \\
4 & $\mathrm{O}_{0} \mathrm{G}_{0} \mathrm{Z}_{1}$ & $\mathrm{G}_{0}$ & $\mathrm{O}_{0} \mathrm{G}_{0} \mathrm{Z}_{1}$ \\
3 & $\mathrm{O}_{1} \mathrm{Z}_{2}$ & $\mathrm{O}_{2} \mathrm{G}_{2}$ & $\mathrm{O}_{2} \mathrm{G}_{2} \mathrm{Z}_{1}$ \\
2 & $\mathrm{O}_{2} Z_{0}$ & $\mathrm{O}_{0}$ & $\mathrm{O}_{1} \mathrm{Z}_{1}$ \\
1 & $\mathrm{O}_{0} \mathrm{Z}_{0}$ & $\mathrm{O}_{2}$ & $\mathrm{O}_{2} \mathrm{Z}_{1}$ \\
\hline
\end{tabular}

which increases $\%$ l by about $5 \%$. The two state model $\mathrm{O}_{2}: \mathrm{O}_{0}$ is equivalent to the variable based model, $\mathrm{O}$, since $\mathrm{df}(\mathrm{O})=2$. The third state selected (model \#4) was $\mathrm{O}_{2} \mathrm{G}_{2}$, indicating an epistatic interaction affecting KIF1B transcription involving a SNP $(O)$ in that gene and a SNP $(G)$ in a different gene, OLA1, but this increases $\%$ only by about $2 \%$, which undermines confidence in the reality of this epistatic effect. Since k-systems analysis is non-statistical, these findings can only be validated by tests in new data.

Step 2.2: Table 13 shows the u-systems analysis of this data. State-based model \#3, namely OG:Z:O $\mathrm{O}_{2}: \mathrm{O}_{1} \mathrm{Z}_{1}$ is equivalent to variable-based $u$-systems model OG:OZ, since $\mathrm{O}_{0} \mathrm{Z}_{1}$ is determined by the known states, $\mathrm{O}_{2} \mathrm{Z}_{1}$ and $\mathrm{O}_{1} \mathrm{Z}_{1}$ (because $Z_{1}$ is known from the $Z$ component) and knowing all $\mathrm{O}_{\mathrm{j}} \mathrm{Z}_{1}$ probabilities determines also the $\mathrm{O}_{\mathrm{j}} \mathrm{Z}_{0}$ probabilities (because $\mathrm{O}$ is known from $\mathrm{OG}$ ). Not surprisingly, therefore, the reconstructed function for this u-systems model is identical to that of the k-systems model \#3, O. As in state-based k-systems analysis, the third added state, $\mathrm{O}_{2} \mathrm{G}_{2} \mathrm{Z}_{1}$, posits an epistatic interaction, increasing $\% 1$ by $4.6 \%$, a slightly greater increase than observed in the k-systems analysis. This added state is in fact the same as what is added in the k-systems search, since $\mathrm{O}_{2} \mathrm{G}_{2} \mathrm{Z}_{1}$ determines also $\mathrm{O}_{2} \mathrm{G}_{2} \mathrm{Z}_{0}$ (because $\mathrm{O}_{2} \mathrm{G}_{2}$ is known from the $O G$ component). Again, not surprisingly, the function reconstructed for this $\mathrm{u}$-systems model is identical to that of $\mathrm{k}$-systems model $\# 4, \mathrm{O}: \mathrm{O}_{2} \mathrm{G}_{2}$. Beyond $\Delta \mathrm{df}=3$, however, $\mathrm{u}$ - and k-analyses diverge, but since $\mathrm{R}^{2}$ for $\mathrm{df}=3$ models is already 0.985, more complex models are not included in Table $\mathbf{1 0}$. The states added in b-, k-, and $\mathrm{u}$-analyses are listed in Table $\mathbf{1 4}$.

In summary, b-systems analysis, both variable- and state-based, suggests an epistatic effect between $O$ and $G$, supported by incremental significance of the models, but k-systems analysis - and perhaps also u-systems analysis casts some doubt upon this. Since $\mathrm{k}$ - and u-systems analyses treat function values exactly, and in state-based models with great precision, their implications carry some weight. The point of these analyses, however, is not to ascertain whether there is an OG epistatic effect on KIF1B gene expression or not. The point is merely to illustrate the methodology of this continuous RA approach as applied to gene expression data; specifically to show that $\mathrm{k}$ - and $\mathrm{u}$-systems approaches can reconstruct functions more accurately than the b-systems approach. A secondary purpose here is to demonstrate that state-based RA is more powerful than variable-based $\mathrm{RA}$, which is strikingly shown by the HY analyses but also evidenced in the KIF1B analyses.

\section{Discussion \& Conclusions}

This paper demonstrates the use of RA to analyze continuous functions, as opposed to frequency or probability distributions, and advances the methods for doing so. In tests on a simple function used in an earlier study on classifying epistasis [21] and on real data [22], k-systems and u-systems analyses yield similar but slightly different results, and have comparable modeling efficacy. The relative merits of these two nonstandard RA approaches to decomposing functions, and their suitability for different data contexts, need to be explored further. In future software development, parallelization of the software will make possible the application of RA to GWAS. After this is done, the number of SNPs that RA can analyze will depend on the type of model. For loopless models, which have closed form solutions, 100,000s of SNPs could be considered; for variable-based models with loops, which require iterative methods, perhaps 1000s (certainly 100s); for state-based models, perhaps 100s (certainly 10s).

Since RA can model epistasis in frequency (and probability) distributions $[\mathbf{1 , 2}]$, and also detect $L D$, and since $k$ - and u-systems variants of RA can be usefully applied also to functions obtained in studies of gene expression, RA 
constitutes a flexible and powerful modeling methodology for the study of epistasis. A variety of other approaches have been applied to modeling epistasis in gene expression [2328]. Comparing RA to other approaches is beyond the scope of this paper, and will be the subject of future studies, but advantages of RA over some other methods can be stated briefly: (i) RA can be used for both nominal data and continuous function applications; this allows one to work within a single mathematical/computational framework, while some other methods are specific to only one these two applications; (ii) RA has three levels of refinement - coarse (variable-based models without loops), fine (variable-based models with loops), and ultra-fine (state-based models); this allows one to move smoothly within a single framework from broad search, e.g., GWAS, involving very many variables to ultrafine analysis that focuses on only a few, while some other methods (e.g., [21]) are specific to one of these situations; (iii) RA explicitly considers the space of possible models in its use of hypergraphs, and is thus especially designed for exploratory searches, while some other methods are primarily confirmatory, and require the user to specify the models to be considered; (iv) Even where RA overlaps with - and to the extent of the overlap is obviously not superior to - other methods, it has distinctive features, so it complements these other methods (see, e.g., the discussion of RA vs. Bayesian networks and other graphical models in [2]).

\section{Competing interests}

The authors declare that they have no competing interests.

\section{Authors' contributions}

$\mathrm{MZ}$ provided the main contribution to this work: drawing on pioneering work of Bush Jones and in collaboration with Michael Johnson (his graduate student), he developed the theory for applying RA to continuous functions, did the reported calculations on the data, and wrote the paper. JF programmed RA in the software package (OCCAM) used as the primary methodological instrument in this research. BW provided the real SNP data, did the initial study that applied RA to gene expression data, and helped in the interpretation of results.

Authors' information

$\mathrm{MZ}$ is one of the principal Reconstructability Analysis theorists today; his many years of work on RA is documented in his web page, http://www.pdx.edu/sysc/research-discrete-multivariatemodeling. He has also directed the development of the RA software package, OCCAM, since its inception. JF, a graduate student in the Systems Science Graduate Program, has been the OCCAM programmer for a number of years. BW is a genomics researcher at the Oregon Health Sciences Researcher; she took MZ's RA course several years ago, and in preliminary studies showed that RA can detect epistasis in gene expression data.

\section{Acknowledgements and funding}

This project was supported in part by the Oregon Tax CheckOff Alzheimer's Research Fund administered by the Layton Aging \& Alzheimer's Disease Center in collaboration with the Oregon Partnership for Alzheimer's Research.

\section{Publication history}

Received: 29-May-2012 Revised: 21-June-2012

Re-revised: 17-Aug-2012 Accepted: 28-Aug-2012

Published: 03-Oct-2012

\section{References}

1. Shervais, S. et al.: Reconstructability analysis as a tool for identifying gene-gene interactions in studies of human diseases. Stat Appl Genet Mol Biol 2010, 9(1):Article18. | Article | PubMed

2. Zwick, M.: Reconstructability analysis of epistasis. Ann Hum Genet 2011, 75(1):157-171. | Article | PubMed

3. Klir, G.: Identification of Generative Structures in Empirical Data. Internat. J. Gen. Sys 1976, 3:89-104. | Article

4. Klir, G.: (1985). The Architecture of Systems Problem Solving. New York: Plenum Press.

5. Krippendorff, K.: An Algorithm for Identifying Structural Models of Multivariate Data. Internat J Gen Sys 1981, 7(1):63-79. | Article

6. Krippendorff, K. (1986). Information Theory. Beverly Hills: Sage.

7. Klir, G.: Reconstructability Analysis: An Offspring of Ashby's Constraint Theory. Systems Research 1986, 3(4): 267-271. | Article

8. Zwick, M.: Wholes and Parts in General Systems Methodology. The Character Concept in Evolutionary Biology 2001, 237-256. New York: Academic Press. I Article

9. Zwick, M.: An Overview of Reconstructability Analysis. Kybernetes 2004, 33(5/6):877-905. | Article

10. Zwick, M.: Reconstructability Analysis With Fourier Transforms. Kybernetes 2004, 33(5/6):1026-1040. | Article

11. Willett, K. et al.: A Software Architecture for Reconstructability Analysis. Kybernetes 2044, 33(5/6):997-1008. | Article

12. Fusion, J., Willett, K. and Zwick, M. (2011). OCCAM: A Reconstructability Analysis Program. | Article | PubMed Abstract | PubMed Full Text

13. Johnson, M.: State-Based Systems Modeling: Theory, Implementation, and Applications. 2005, 219:3183756. PhD Dissertation, Portland State University.

14. Zwick, M.: Information-Theoretic Mask Analysis of Rainfall Time-Series Data. Advances in Systems Science and Applications, 2005, 1:154-159. | Article

15. Wilmot, B., Zwick, M., and McWeeney, S. (2008). Reconstructability Analysis Detects Genetic Variation Associated with Gene Expression. 12th QTL-MAS Workshop in Computational Genetics, Uppsala, Sweden, May 15-16. | Article | PubMed Abstract | PubMed Full Text

16. Myers, A. J. et al.: A survey of genetic human cortical gene expression. Nat Genet 2007, 39(12):1494-1499. | Article I PubMed

17. Jones, B.: Reconstructability Analysis for General Functions. Internat. J Gen Sys 1985, 11:133-142. | Article

18. Johnson, M. et al.: State-Based Reconstructability Modeling For Decision Analysis. In Proceedings of The World Congress of the Systems Sciences and ISSS 2000, (eds. J.K. Allen \& J.M. Wilby), Toronto, Canada: International Society for the Systems Sciences. | Article

19. Jones, B.: K-systems Versus Classical Multivariate Systems. Internat. J Gen Sys 1985, 12(1):1-6. | Article

20. Gouw, D. et al.: Using K-systems Theory to Compute True Main and Interaction Effects in a Design of Experiments. Cybernetics and Systems: An International Journal, 1995, 26(4):481-498. | Article

21. Hallgrimsdottir, I. B. \& Yuster, D. S.: A complete classification of epistatic two-locus models. BMC Genet 2008, 9:17. | Article | PubMed Abstract I PubMed Full Text

22. Wilmot, B. (2008). Association of SNPs and Gene Expression Using Reconstructability Analysis: A Proof of Principle. Oregon Clinical + Translational Research Institute unpublished report, April 8, 2008. I Article | PubMed Abstract | PubMed Full Text

23. Culverhouse, R., Klein, T. \& Shannon, W.: Detecting epistatic interactions contributing to quantitative traits. Genet Epidemiol 2004, 27(2):141152. Genet. Epidemiol. 27(2):141-52. | Article | PubMed

24. Wang, T. \& Zeng, Z. B.: Models and partition of variance for quantitative trait loci with epistasis and linkage disequilibrium. $B M C$ Genet 2006, 7:9. | Article | PubMed Abstract | PubMed Full Text

25. Jannink, J. L.: Identifying quantitative trait locus by genetic background interactions in association studies. Genetics 2007, 176(1):553-561. | 
Zwick et al. Journal of Molecular Engineering \& Systems Biology 2012, http://www.hoajonline.com/journals/pdf/2050-1412-1-4.pdf

Article | PubMed Abstract | PubMed Full Text

26. Wang, T. \& Zeng, Z. B.: Contribution of genetic effects to genetic variance components with epistasis and linkage disequilibrium. $B M C$ Genet 2009, 10:52. | Article | PubMed Abstract | PubMed Full Text

27. Michaelson, J. J., Alberts, R., Schughart, K. \& Beyer, A.: Data-driven assessment of eQTL mapping methods. BMC Genomics 2010, 11:502. | Article | PubMed Abstract | PubMed Full Text

28. Zhang, Y., Jiang, B., Zhu, J. \& Liu, J. S.: Bayesian models for detecting epistatic interactions from genetic data. Ann Hum Genet 2011, 75(1):183-193. | Article | PubMed

\section{Citation:}

Zwick M, Fusion J and Wilmot B: Reconstructability of Epistatic Functions. journal of Molecular

Engineering and Systems Biology 2012, 1:4.

http://dx.doi.org/10.7243/2050-1412-1-4 